\title{
Pulsed Doppler echocardiographic assessment of portal venous flow patterns in patients after the Fontan operation
}

Departments of Radiology, Paediatrics, and Surgery, Osaka University Medical School, Fukushima Fukushimaku, Osaka, Japan

J Arisawa

S Morimoto

$\mathrm{J}$ Ikezoe

H Naitoh

H Yamagami

T Kozuka

T Sano

Y Shimazaki

H Matsuda

Correspondence to: un Arisawa, Department of Radiology, Osaka University Medical School, 1-1-50 Fukushima, Fukushimaku, Osaka 553, Japan

Accepted for publication 15 July 1992

Jun Arisawa, Shizuo Morimoto, Junpei Ikezoe, Hiroaki Naitoh, Hidetoshi Yamagami, Takahiro Kozuka, Tetsuya Sano, Yasuhisa Shimazaki, Hikaru Matsuda

\begin{abstract}
Objective-To assess the effect of the condition of the right heart after the Fontan operation on portal venous flow, and to determine whether the characteristics of portal venous flow were different when there was an atriopulmonary connection with atrial septal closure rather than an atriopulmonary or total cavopulmonary connection with intra-atrial routing.
\end{abstract}

Patients and methods-After the Fontan operation six patients with an atriopulmonary connection (group 1), three patients with an atriosubpulmonary connection (group 2), four patients with intra-atrial routing and an atriopulmonary connection (group 3), and five patients with a total cavopulmonary connection (group 4) were studied by pulsed Doppler echocardiography. The flow signals were recorded for the pulmonary artery, hepatic vein, and intrahepatic portal vein in each patient. Postoperative cardiac catheterisation was performed in 16 of the 18 patients. The Doppler findings were compared with those of 14 controls.

Results-The portal flow was pulsatile in 13 patients and constant in five patients. Reversed flow was shown at or just after the QRS wave after hepatic venous regurgitation in two group 1 patients. The flow signal was interrupted in two group 1 patients and two group 2 patients. Decrease in velocity of flow was recorded in two group 1 patients, one group 2 patient, three group 3 patients, and one group 4 patient. Portal flow was constant in one group 3 patient and four group 4 patients. The pulsatility ratio ranged from -0.46 to 0.49 (mean (SD), $0.03(0.32))$ in the patients from group 1 and 2 , from 0.41 to $0.76(0.54(0.15))$ in group 3, and from 0.70 to $0.80(0.75$ $(0.04))$ in group 4. The ratio in the controls ranged from 0.29 to $0.83(0.61$ $(0 \cdot 13))$. The ratio was significantly lower in groups 1 and 2 than in group 3 (p < $0.01)$, group $4(p<0.01)$, or the controls $(p<0.005)$. There was no significant difference in the ratio between group 3 and group 4 and no correlation between the pulsatility ratio and the cardiac index $(r$ $=0.26)$, mean right atrial pressure $(r=$ $0 \cdot 25)$, or pulmonary vascular resistance $(r=0 \cdot 17)$
Conclusion-The larger hepatic venous regurgitation caused by atrial contraction in patients with an atriopulmonary connection correlated with the higher portal pulsatility and a total cavopulmonary connection reduced portal pulsatility.

(Br Heart f 1993;69:41-46)

Blood flow in the normal portal vein in adults is almost continuous except for a velocity change due to breathing. ${ }^{1}$ The pulsatility of the Doppler signals was increased in adults with congestive heart failure caused by mitral disease or cardiomyopathy. ${ }^{2}$ Lessening of the velocity or reversed flow was shown during ventricular diastole after hepatic venous regurgitation due to tricuspid regurgitation. ${ }^{2}$ In patients after the Fontan operation, the right atrial mean pressure is raised, and right atrial contraction accelerated the rate for one of the two peaks of pulmonary arterial flow, ${ }^{34}$ but it also produced reversed flow in the inferior vena cava and hepatic vein. One of the aims of this study was to use pulsed Doppler echocardiography to assess the effect of the right heart condition on the portal venous flow pattern.

A total cavopulmonary connection with an intra-atrial tunnel can be used to connect the inferior vena cava and pulmonary artery in any atrioventricular arrangement. ${ }^{5}$ The larger part of the atrial chamber remains at low pressure, and the risk of arrhythmias is reduced in this procedure. The intra-atrial conduit has little or no contraction and this reduces regurgitation from the atrium into the vena cava and hepatic vein. The other aim of this study was to assess the difference in the effects of an atriopulmonary connection and a total cavopulmonary connection on the hepatic circulation.

\section{Patients and methods}

PATIENTS

We studied 18 consecutive patients who underwent echocardiographic examination for assessment of the cardiac condition after the Fontan operation between August 1988 and August 1991. Table 1 shows the diagnosis and surgical procedure in each patient. There were seven patients with tricuspid atresia, two patients with pulmonary atresia, eight patients with a single ventricle, and one patient with 
Table 1 Diagnosis and operative procedures in 18 patients

\begin{tabular}{cllc}
\hline Patient No & Diagnosis & Surgical procedure & Age at operation (y) \\
\hline Group 1: & TA & ASD, RA-PA & 3 \\
1 & TA & ASD, RA-PA & 4 \\
2 & TA PS & ASD, RA-PA & 9 \\
3 & TA PS & ASD, RA-PA & 8 \\
4 & PA & ASD, RA-PA & 8 \\
5 & SV (LV type) PS & ASD, RA-PA & 12 \\
6 & TA & ASD, RA-SPRV & 4 \\
Group 2: & TA PS & ASD, RA-SPRV & 3 \\
7 & PA & ASD, RA-SPRV & 5 \\
8 & & & 8 \\
9 & CAVV SV (RV type) PS & IAR, RA-PA & 5 \\
Group 3: & IAR, RA-PA & 12 \\
10 & SA (RV type) PS & IAR, RA-PA & 7 \\
11 & IAR, RA-PA, LSVC-LPA & \\
12 & RAI SV (RV type) PS & IAR, TCPC & 5 \\
Group 4: & SV (RV type) PS & IAR, TCPC & 6 \\
14 & IAR, TCPC & 4 \\
15 & SV (LV type) PS & IAR, TCPC & 4 \\
16 & SV (LV type) & IAR, TCPC & 4 \\
17 & LAI LAVVA PS & TA PS & \\
\hline
\end{tabular}

ASD, closure of atrial septal defect; CAVV, comon atrioventricular valve; IAR, intra-atrial routing; LAI, left atrial isomerism; LSVC-LPA, connection of left superior vena cava to left pulmonary artery; LV, left ventricle; PA, pulmonary atresia; PS, pulmonary stenosis; RA-PA, connection of right atrium to pulmonary artery; RA-SPRV, connection of right atrium to subpulmonary right ventricle; RAI, right atrial isomerism; RV, right ventricle; SV, single ventricle; TA, tricuspid atresia; TCPC, total cavopulmonary connection.

left atrioventricular valve atresia. The type of single ventricle was determined by angiocardiography and echocardiography. Three patients had a single ventricle of the left ventricular type, which is characterised by a smooth trabecular architecture and an anteriorly positioned rudimentary chamber connected to a great vessel. Five patients had a single ventricle of the right ventricular type which is characterised by a rough trabeculation and a posteriorly positioned rudimentary chamber with no ventriculoarterial connection. Two patients with a single ventricle also had right atrial isomerism. In the patient with left atrioventricular valve atresia, the ventricle was shown echocardiographically to have no rudimentary chamber and the trabeculation was rough. This patient had left atrial isomerism.

The age at the Fontan operation ranged from three to 12 years (mean (SD) 6.4 (1.9)).
The patients were divided into four groups according to the surgical procedure. Group 1 consisted of six patients who underwent closure of an atrial septal defect for atrial partition and the creation of a connection from the right atrium to the pulmonary artery. Group 2 consisted of three patients who underwent closure of an atrial septal defect and creation of a connection from the right atrium to the subpulmonary right ventricle. In these patients, the connection from the right atrium to the right ventricle was performed with a xenopericardium patch without a valve, and postoperative angiocardiography showed no obstructive lesion from the right atrium to the pulmonary artery. Group 3 consisted of four patients who underwent intra-atrial routing with an intra-atrial conduit and creation of a connection from the right atrial appendage to the pulmonary artery. Group 4 consisted of five

Table 2 Postoperative catherisation data and pulsatility ratio of portal vein

\begin{tabular}{|c|c|c|c|c|c|c|}
\hline \multirow[b]{2}{*}{ Patient No } & \multicolumn{5}{|c|}{ Postoperative catheterisation data } & \multirow{2}{*}{$\begin{array}{l}\text { Pulsatility } \\
\text { ratio }\end{array}$} \\
\hline & $m R A P$ & $m P A P$ & $m P A W P$ & $P V R$ & $C I$ & \\
\hline \multicolumn{7}{|l|}{ Group 1: } \\
\hline 1 & 19 & 18 & 12 & $2 \cdot 5$ & $2 \cdot 4$ & -0.46 \\
\hline 2 & 12 & 10 & 2 & $2 \cdot 7$ & 4.9 & -0.40 \\
\hline 3 & 20 & 20 & 7 & $5 \cdot 6$ & $2 \cdot 1$ & 0.38 \\
\hline 4 & 16 & 16 & 6 & $4 \cdot 7$ & $2 \cdot 1$ & 0.00 \\
\hline 5 & 17 & 17 & 4 & & $2 \cdot 1$ & 0.49 \\
\hline 6 & 19 & 17 & 4 & & & 0.00 \\
\hline \multicolumn{7}{|l|}{ Group 2: } \\
\hline 7 & 11 & 10 & 3 & $2 \cdot 3$ & $3 \cdot 1$ & 0.00 \\
\hline 8 & 9 & 9 & 7 & $2 \cdot 0$ & $2 \cdot 0$ & 0.00 \\
\hline 9 & 14 & 14 & 4 & $3 \cdot 8$ & $2 \cdot 1$ & $0 \cdot 25$ \\
\hline \multicolumn{7}{|l|}{ Group 3: } \\
\hline $\begin{array}{l}10 \\
11\end{array}$ & 18 & 16 & 11 & $2 \cdot 7$ & 1.9 & $\begin{array}{l}0.50 \\
0.41\end{array}$ \\
\hline 12 & 8 & 8 & 3 & $2 \cdot 3$ & $2 \cdot 2$ & 0.51 \\
\hline 13 & 13 & 12 & 6 & $3 \cdot 6$ & 1.7 & $0 \cdot 76$ \\
\hline \multicolumn{7}{|l|}{ Group 4: } \\
\hline 14 & 16 & 16 & 7 & $2 \cdot 9$ & $4 \cdot 5$ & $0 \cdot 70$ \\
\hline 15 & 12 & 12 & 6 & $4 \cdot 2$ & $2 \cdot 0$ & 0.74 \\
\hline 16 & 16 & 16 & 3 & $7 \cdot 2$ & $2 \cdot 7$ & 0.76 \\
\hline 17 & 17 & 17 & 8 & $4 \cdot 7$ & 2.5 & 0.75 \\
\hline 18 & - & - & - & - & - & $0 \cdot 80$ \\
\hline
\end{tabular}

Postoperative catheterisation had not been performed in patient 11 and 18 . Left to right shunt $(\mathrm{Qp} / \mathrm{Qs}=1.94)$ was found Postoperative catheterisation had not been performed in patient 11 and 18 . Lef to monary artery pressure; mPAWP, mean through the reopened pulmonary artery in patient 6 . Cl, cardiac index; mPAP, mealmonary vascular resistance. 

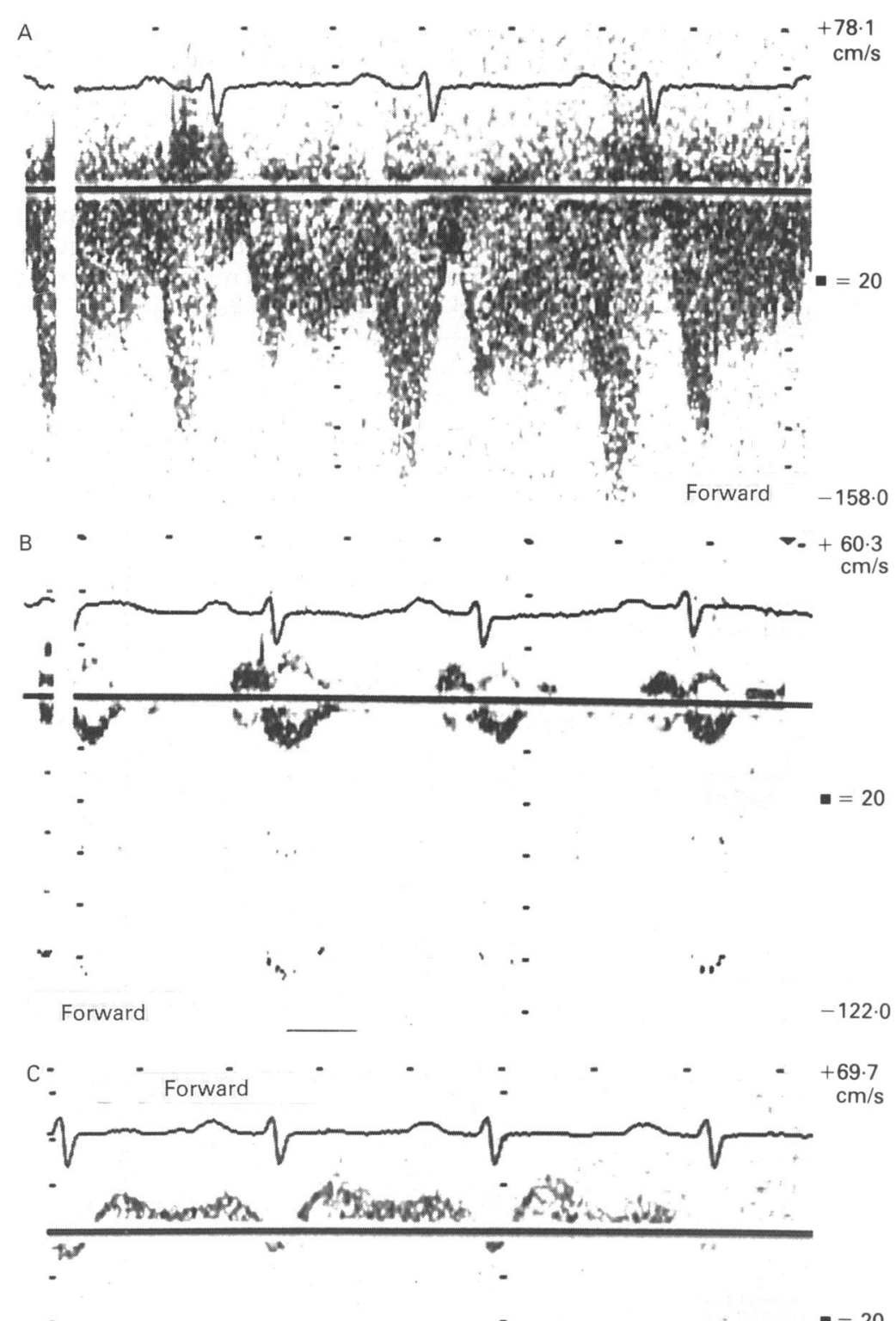

$=20$

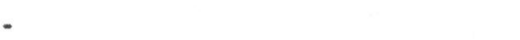

-

Figure 1 Pulsed Doppler recording from pulmonary artery $(A)$, hepatic vein $(B)$, and portal vein $(C)$ in a three year old male patient (case 2) one week after Fontan operation with atrial septal closure and an atriopulmonary connection. Pulmonary artery showed two flow peaks: late diastolic and systolic to early diastolic. The peak flow velocity of late diastole was higher than the other. Hepatic venous flow had a regurgitant peak after the $P$ wave. Portal venous flow showed regurgitant flow at the $Q R S$ wave and the pulsation was biphasic.

\section{DOPPLER ECHOCARDIOGRAPHY}

The Doppler echocardiographic study was performed with a Hewlett-Packard 77020AC ultrasound system from nine days to 66 months (mean 728 (173) days) after the Fontan operation. The Doppler signals were simultaneously recorded on videotape and on imaging film with a lead II electrocardiogram. The pulsed Doppler flow signal was recorded at three sites in the right heart: the pulmonary artery or right atrium to pulmonary artery conduit by a precordial approach, the hepatic vein by a subcostal approach, and the portal vein by a subcostal or right intercostal approach. To record portal flow the posterior segmental branch of the right lobe was first distinguished from the epigastrium. If recording was impossible, the signal was recorded in the anterior segmental branch of the right lobe or right lobe branch by right intercostal scanning. In two patients with asplenia the Doppler signals were taken from one of the intrahepatic branches. The portal flow signal was recorded at the end of normal expiration or with the breath held at normal expiration if possible. A peak to peak pulsatility ratio (minimum frequency shift/maximum frequency shift) of the portal Doppler wave form was calculated for each patient. ${ }^{2}$ The pulsatility ratio decreases as the flow becomes more pulsatile.

\section{CARDIAC CATHETERISATION}

Cardiac catheterisation was performed in 16 of the 18 patients for routine evaluation of the postoperative state at 21 days to 22 months (mean 226 (60) days) after the Fontan operation. The interval between catheterisation and Doppler echocardiographic study was less than one month in five patients and less than two months in two patients. In the other nine patients, catheterisation preceded the echocardiography by 12 64 months. The patients underwent cardiac catheterisation under mild sedation (pethidine hydrochloride and hydroxyzine). Pressures were recorded through a well flushed, fluid filled catheter connected to a Statham strain gauge (P23Db or P23ID). The cardiac output was measured by the dye dilution method.

\section{STATISTICAL ANALYSIS}

Wilcoxon signed rank tests were used to determine whether there was any difference in the pulsatility ratio between the control and patient groups. A $p$ value $<0.05$ was considered significant. The data are presented as the mean (SD).

patients who underwent intra-atrial routing with an intra-atrial conduit and total cavopulmonary connection.

\section{CONTROL GROUP}

The control group consisted of 14 consecutive subjects (aged from one to 13 years (mean 6.2 (3.8)) who underwent echocardiographic examination between July 1989 and September 1989 because of a heart murmur: they were found to be normal.

\section{Results}

PULSED DOPPLER FLOW PATTERN

The pulmonary artery showed biphasic pulsatility in all the group 1 and group 2 patients, in three group 3 patients, and two group 4 patients. One flow peak began at the beginning of the $P$ wave and another just after the QRS wave in these patients (figs 1 


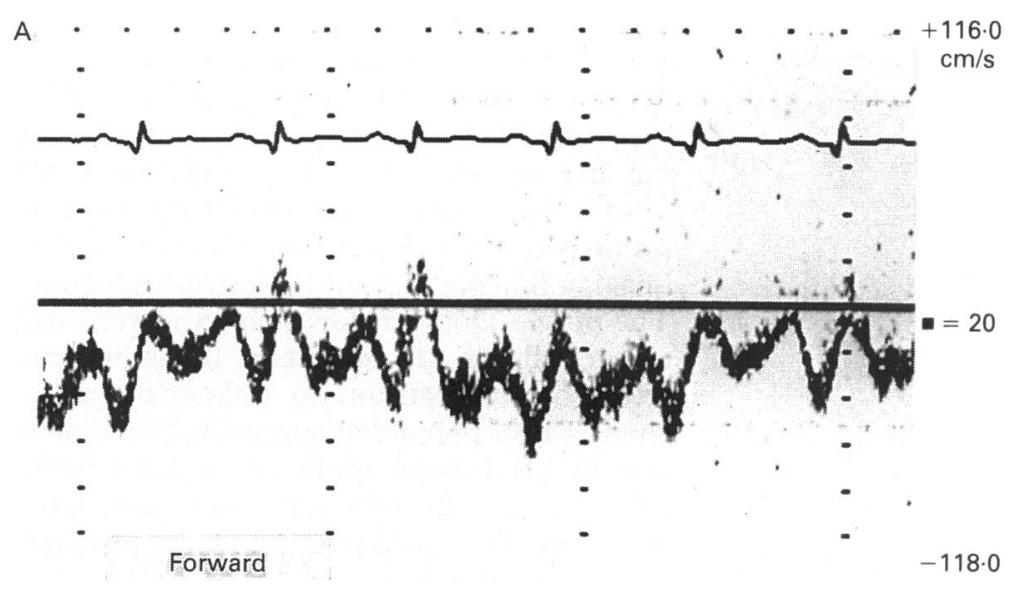

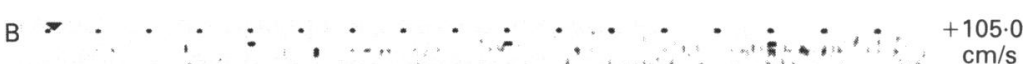
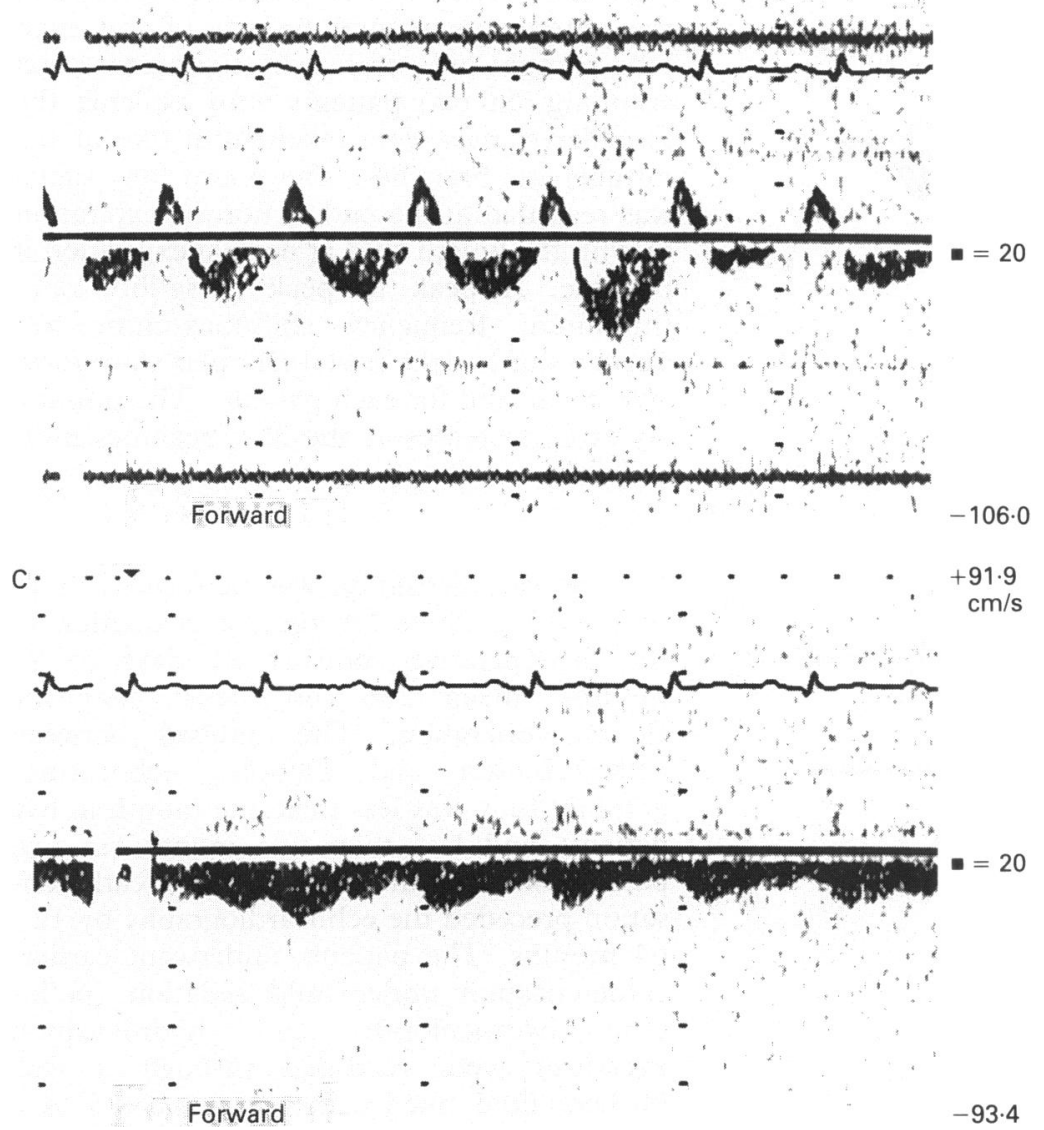

Figure 2 Pulsed Doppler recording from pulmonary artery $(A)$, hepatic vein $(B)$, and portal vein $(C)$ in a six year old female patient (case 11) one month after Fontan operation with intra-atrial routing and an atriopulmonary connection. Pulmonary artery showed two flow peaks: late diastolic and systolic to early diastolic. The peak flow velocity of late diastole was higher than the other. Regurgitant flow was recorded at atrial systole in the hepatic vein. Decrease in portal flow velocity was shown at the QRS wave.

and 2). One small flow peak was found just after the flow peak that began after the QRS in one other group 3 patient whose $R R$ interval was $944 \mathrm{~ms}$. The maximum velocity of the peak at atrial systole was higher than that at ventricular systole in groups 1,2 , and 3 (figs 1 and 2). In two group 4 patients the maximum velocity at atrial systole was lower than that at ventricular systole (fig 3). A constant pulmonary arterial flow was recorded in three other group 4 patients. The hepatic vein showed reversed flow just after the $P$ wave in all the group 1 and 2 patients, three group 3 patients, and four group 4 patients (figs 1,2 and 3). In another two patients, one in group 3 and one in group 4, the flow was only interrupted. The portal flow was pulsatile in 13 patients and constant in five patients. Flow was reversed at or just after the QRS wave after hepatic venous regurgitation in two $\underline{\underline{T}}$ patients of group 1 (fig 1). The flow signal was interrupted after hepatic venous regurgitation in two group 1 patients and two group 2 patients (fig 4). A decrease in the flow velocity after the hepatic regurgitant flow was $\overrightarrow{\vec{\sigma}}$ recorded in two group 1 patients, one group $\overline{0}$ 2 patient, three group 3 patients, and one group 4 patient (fig 2 ). A constant portal flow $\frac{\bar{\rho}}{\frac{1}{\sigma}}$ was recorded in one group 3 patient and four $\stackrel{\mathbb{\Omega}}{\varrho}$ group 4 patients (fig 3 ). The pulsation was monophasic in one patient, biphasic in $11 \overrightarrow{0}$ patients, and triphasic in one patient whose pulmonary arterial flow was triphasic.

PULSATILITY RATIO OF THE PORTAL VEIN

Table 2 shows the catheterisation data and 8 pulsatility ratio of the portal vein for each $\vec{A}$

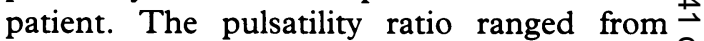
-0.46 to $0.49(0.03(0.32))$ in the group 1 은 and 2 patients, from 0.41 to $0.76(0.54 \vec{c}$ $(0 \cdot 15))$ in group 3 patients, and from 0.70 to $0.80(0.75(0.04))$ in group 4 patients (fig 5). The ratio in the controls ranged from 0.29 to $0.83(0.61(0.13))$ (fig 5). The ratio was sig- $\overrightarrow{0}$ nificantly lower in group 1 and group 2 than in group $3(p<0.01)$, group $4(p<0.01)$, or the controls $(p<0.005)$. There was no significant difference in the ratio between group 3 and group 4. There was no correlation between the pulsatility ratio and the cardiac $\mathscr{Q}$ index $(r=0 \cdot 26)$, mean right atrial pressure $(r \overrightarrow{\overrightarrow{0}}$ $=0.25)$, or pulmonary vascular resistance $(r$ $=0 \cdot 17$ ).

\section{Discussion}

In healthy people the hepatic venous flow shows biphasic pulsatility and reversal at right 3 . atrial contraction and at ventricular end systole. ${ }^{6}$ It does not, however, affect the portal venous flow pattern, and the portal flow is 0 almost constant or shows a mild pulsatility. ${ }^{1}$ This phenomenon is explained by the hepatic venous or sinusoidal reserve. Severe tricuspid regurgitation results in the disappearance of forward flow and regurgitation during systole in the hepatic vein. ${ }^{7}$ Portal flow pulsation 5 related to congestive heart failure or tricuspid regurgitation has been reported by several groups. ${ }^{128}$

In our patients after the Fontan operation: with atrial septal closure and atriopulmonary connection pulsed Doppler showed increased pulsatility of the portal venous flow. Blood flow in the right heart is changed by the absence of the ventricular chamber between the right atrium and the pulmonary artery in $\frac{0}{0}$ patients after the Fontan operation. The absence of ventricular contraction and the $\frac{\bar{a}}{2}$ absence of valves increase the right atrial mean pressure, and the presence of right atrial contraction without valvar competence between the inferior vena cava and the pulmonary artery influences the hepatic and por- 
A

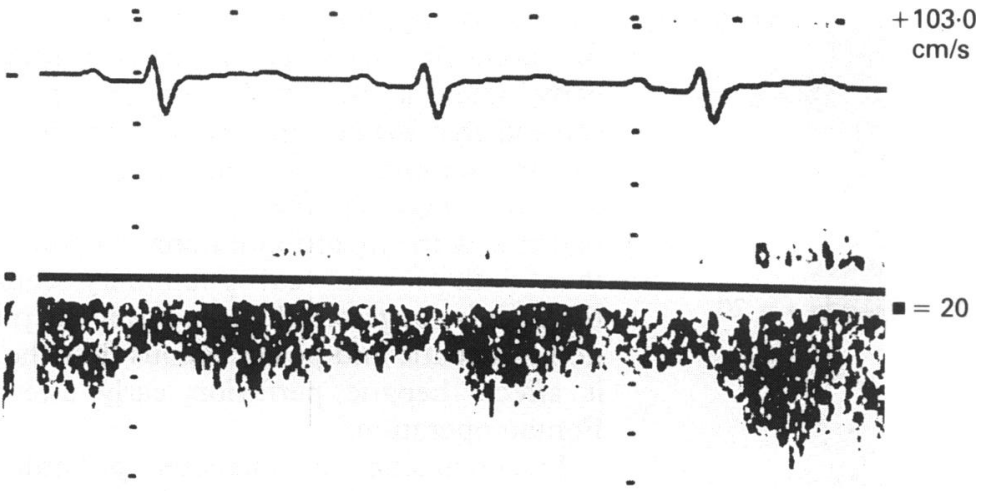

Forward

B
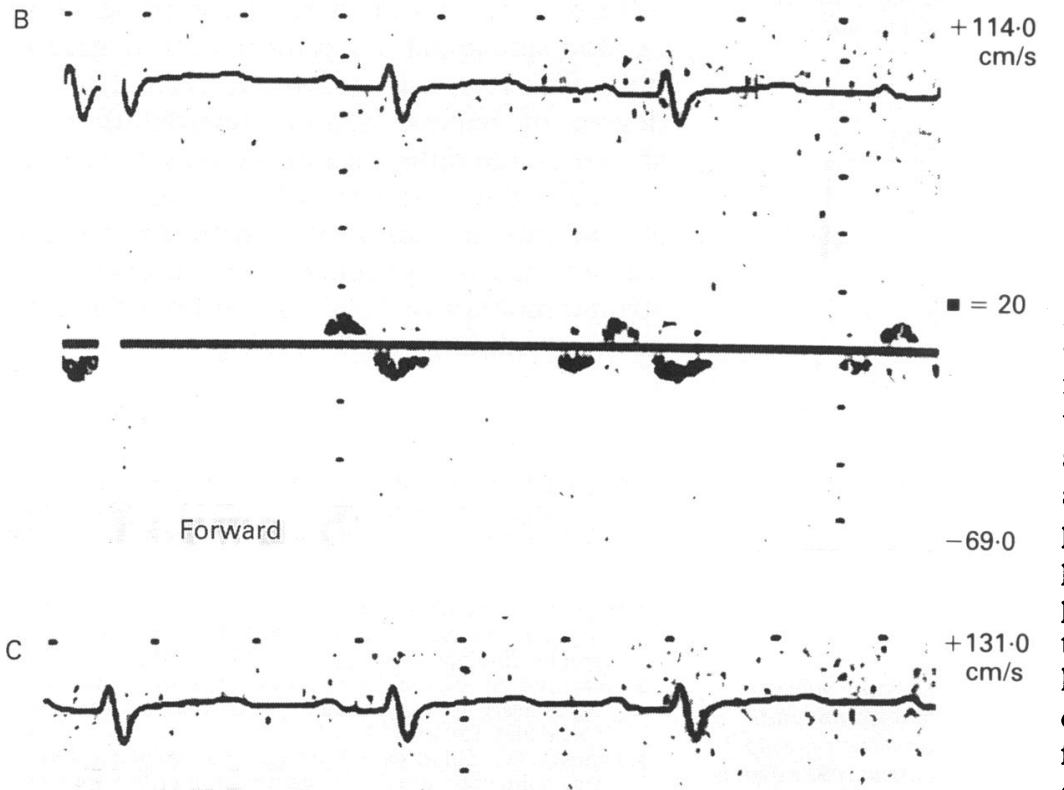

Forward

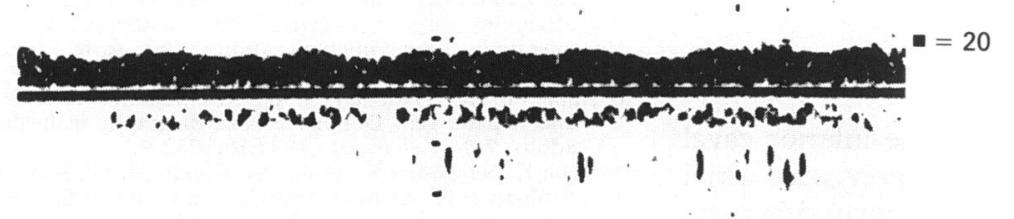

$-79 \cdot 6$

Figure 3 Pulsed Doppler recording from pulmonary artery $(A)$, hepatic vein $(B)$, and portal vein (C) in a six year old boy (case 18) two weeks after Fontan operation with total cavopulmonary connection. Pulmonary artery showed continuous flow with mild biphasic pulsation. The peak flow velocity of late diastole is lower than the other. Regurgitant flow was recorded at atrial systole in the hepatic vein. Constant flow was recorded in the portal vein. venous forward flow. The pulmonary artery has another forward flow peak late in ventricular systole. This is considered to be caused by the passive effect of pulmonary venous flow derived from left atrial relaxation. ${ }^{49}$ The portal venous flow has another phase of lessening that is not caused by the atrial contraction, and therefore the biphasic flow pattern is considered to correspond to the biphasic flow pattern of the pulmonary artery.

In patients after total cavopulmonary connection, Doppler echocardiographic study of the pulmonary artery shows a continuous flow pattern with slight or no pulsation. In the cases with mild pulsation, the peak at atrial systole is lower than the peak that begins late in ventricular systole. This shows that the intra-atrial conduit has no pump function. The reduced pulsation in pulmonary arterial flow may be a disadvantage to the pulmonary circulation. An animal model of atriopulmonary connection without intra-atrial routing did not show that the atrium was actually pumping the systemic venous return into the pulmonary artery. ${ }^{10}$ The catheterisation data of our series showed no significant difference in the mean pulmonary arterial pressure or the cardiac index between patients with an atriopulmonary connection without intra-atrial routing and patients with a total cavopulmonary connection. Therefore, the pulmonary circulation is well preserved in patients with a total cavopulmonary connection for a short period after operation. The long-term result might be affected by the development of a pulmonary arteriovenous fistula. A pulmonary arteriovenous fistula has caused a significant drop in systemic oxygen saturation in patients after Glenn's shunt operation or total cavopulmonary shunt operation. ${ }^{11}$ The maximum follow up period of our series of total cavopulmonary connection was one year, and during that short period there was no evidence of systemic desaturation. A long-term follow up study would prove whether a pulmonary arteriovenous fistula develops in patients after total cavopulmonary connection.

A significant difference in the pulsatility ratio was shown between the patient groups. The patients who underwent intra-atrial routing with a conduit and atriopulmonary or total cavopulmonary connection showed a significantly higher ratio and so indicated lower pulsatility. By contrast, there was no correlation between the pulsatility ratio and the right atrial mean pressure, cardiac index, or pulmonary vascular resistance. It is not accurate to discuss the correlation of the pulsatility ratio and the catheterisation data, because cardiac catheterisation and Doppler assessment were not simultaneous. There was, however, no significant difference in the right atrial mean pressure, cardiac index, or pulmonary vascular resistance between the groups of patients. Therefore, it is presumed that the pulsatility ratio correlated more significantly with the surgical procedure than the catheterisation data. The baffle or tunnel, right atrial contraction simultaneously accelerates the peak forward flow in the pulmonary artery. ${ }^{3}$ After that event, right atrial relaxation causes rapid hepatic venous and portal 


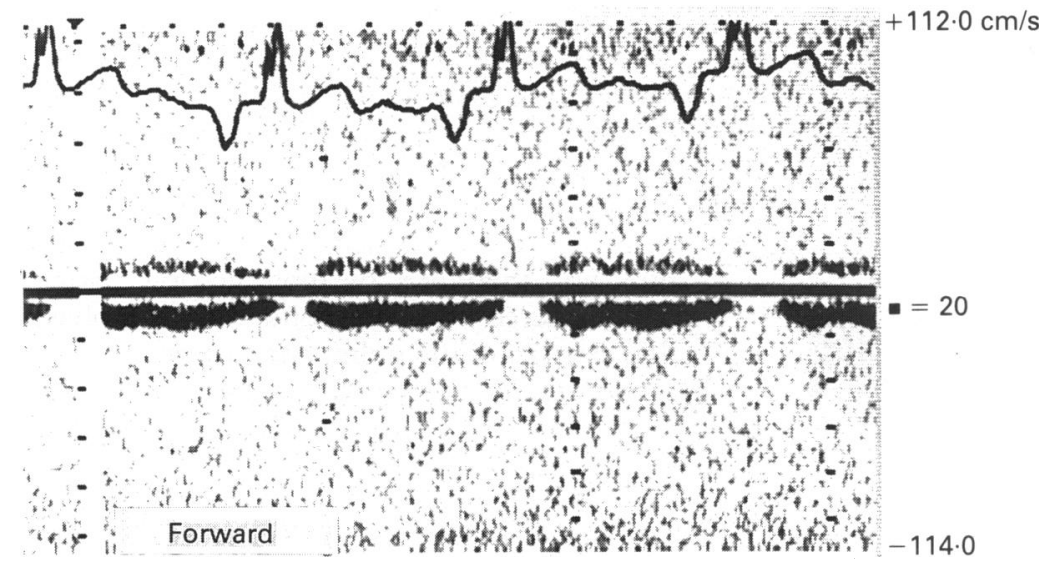

Figure 4 Pulsed Doppler recording from the portal vein in a 12 year old boy (case 4) 57 months after Fontan operation with atrial septal closure and an atriopulmonary connection. Note the interruption of the flow signal at the $Q R S$ wave.

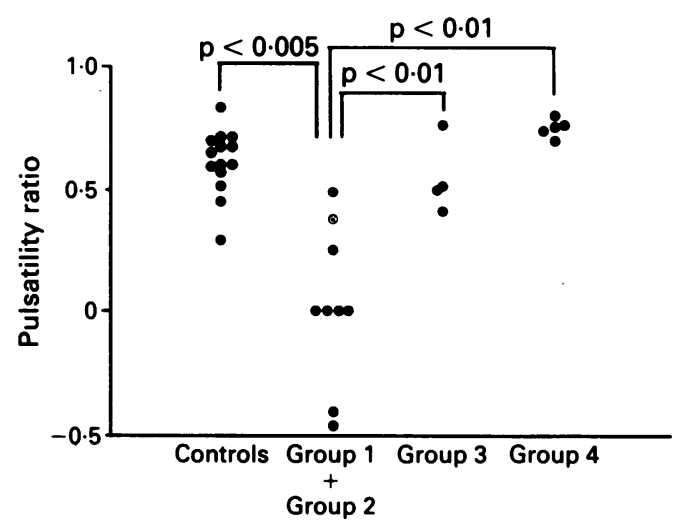

Figure 5 Plot of the pulsatility ratio in normal subjects (controls), in the patients with atrial septal closure and atriopulmonary or atriosubpulmonary connection (group 1 and group 2), in the patients with intra-atrial routing and atriopulmonary connection (group 3), and in the patients with total cavopulmonary connection (group 4). The ratio was significantly lower in group 1 and 2 than in group 3, group 4, or controls.

which was used to connect the inferior caval vein to the pulmonary artery, prevented atrial contraction and resulted in decreased hepatic venous regurgitation, by contrast with the cases of atrial septal closure and an atriopulmonary connection. Therefore, the decreased pulsatility of the portal vein in patients with intra-atrial routing and an atriopulmonary or total cavopulmonary connection is related to the decreased hepatic venous regurgitation. It was then indicated that the hepatic venous regurgitation causes portal venous pulsation under the condition of right heart congestion. The benefit of reduced pulsatility of the hepatic portal venous flow after the total cavopulmonary connection compared with that after the atriopulmonary con- nection is supposed to be protective of liver function. An investigation of liver function early after a modified Fontan operation showed that maximum serum glutamic pyruvic transaminase was higher and hepatic venous oxygen saturation was lower in the patients with an atriopulmonary connection than with a total cavopulmonary connection. ${ }^{12}$ It is suggested that the lower the pulsation of hepatic and portal venous flow the less it affects hepatic perfusion early after the Fontan operation.

In conclusion, the increased pulsatility of the portal vein in patients after the Fontan operation with an atriopulmonary connection indicates that hepatic venous regurgitation influences the portal flow in the presence of hepatic sinusoidal congestion. The degree of pulsatility is considered to be related to the degree of hepatic venous regurgitation, as shown by the difference in the pulsatility ratio between the patients who underwent an atriopulmonary connection with atrial septal closure and the patients who underwent an atriopulmonary or total cavopulmonary connection with intra-atrial routing.

1 Smith HJ, Grottum P, Simonsen S. Ultrasonic assessment of abdominal venous return. I. Effect of cardiac action and respiration on mean velocity pattern, cross-sectional area and flow in the inferior vena cava and portal vein. Acta Radiol 1985;26:581-8.

2 Hosoki T, Arisawa J, Marukawa T, et al. Portal blood flow in congestive heart failure: pulsed Doppler duplex sonographic findings. Radiology 1990;174:733-6.

3 Nakazawa $M$, Nakanishi $T$, Okuda $H$, et al. Dynamics of right heart flow in patients after Fontan procedure. right heart flow in patients
Circulation 1984;69:306-12.

4 DiSessa TG, Child JS, Perloff JK, et al. Systemic venous and pulmonary arterial flow patterns after Fontan's procedure for tricuspid atresia or single ventricle. Circulation 1984;70:898-902.

5 de Leval MR, Kilner P, Gewillig M, Bull C. Total cavopulmonary connection: a logical alternative to atriopulmonary connection for complex Fontan operations. Experimental studies and early clinical experience. F Thorac Cardiovasc Surg 1988;96:682-95.

6 Appleton CP, Hatle LV, Popp RL. Superior vena cava and hepatic vein Doppler echocardiography in healthy adults. $\Im$ Am Coll Cardiol 1987;10:1032-9.

7 Sakai K, Nakamura K, Satomi G, Kondo M, Hirosawa K. Evaluation of tricuspid regurgitation by blood flow pattern in the hepatic vein using pulsed Doppler technique. tern in the hepatic vein using

8 Diebold B, Touati R, Blanchard D, et al. Quantitative assessment of tricuspid regurgitation using pulse Doppler echocardiography. Br Heart $₹$ 1983;50:443-9.

9 Miura T, Matsuda H, Nakano S, et al. Assessment of pulmonary blood flow after total cavopulmonary shunt operation and the modified Fontan procedure for univentricular heart. Fournal of Cardiography 1988;18: 837-44. (In Japanese)

10 Matsuda H, Kawashima Y, Takano H, Miyamoto K, Mori T. Experimental evaluation of atrial function in right atriumpulmonary artery conduit operation for tricuspid atresia. $\mathcal{F}$ Thorac Cardiovasc Surg 1981;81:762-7.

11 McFaul RC, Tajik AJ, Mair DD, Danielson GK, Seward JB. Development of pulmonary arteriovenous shunt after superior vena cava-right pulmonary artery (Glenn) anter superior vena cava-right pulmonary

12 Matsuda H, Arisawa J, Shimazaki Y, Iio M, Kadoba K Sano T, et al. Evaluation of total cavopulmonary connection in modified Fontan operation from hepatic ci culation [abstract]. $\mathcal{F}$ Am Coll Cardiol 1992;19:232. 